\title{
Application of Ubiquitous Devices for Fishery Control of Endangered Species
}

\author{
Rhayane S. Monteiro ${ }^{1}$, Mário W. L. Moreira ${ }^{1}$, Glácio S. Araújo ${ }^{1}$, \\ Joel J. P. C. Rodrigues ${ }^{2,3}$, Victor Hugo C. de Albuquerque ${ }^{4}$ \\ ${ }^{1}$ Instituto Federal de Educação, Ciência e Tecnologia do Ceará (IFCE) \\ Aracati, CE - Brazil \\ ${ }^{2}$ Federal University of Piauí (UFPI), Teresina - PI, Brazil \\ ${ }^{3}$ Instituto de Telecomunicações, Portugal \\ ${ }^{4}$ Universidade de Fortaleza (Unifor), Fortaleza, CE - Brazil \\ \{rhayane.monteiro, mario.wedney, glacio\}@ifce.edu.br \\ joeljr@ieee.org, victor.albuquerque@unifor.br
}

\begin{abstract}
Recognition of fish species is of great importance to marine biology and aquaculture. Ubiquitous devices represent an efficient solution for the preservation of species through the monitoring of fish in the risk of extinction. This approach is essential for endangered population assessment as well as for ecosystem preservation. Several methods have been assessed in these devices to solve the complex task of identifying at-risk of overfishing. As an alternative, the convolutional neural networks (CNNs) represent an accurate method for pattern recognition. Hence, this paper proposes the performance evaluation of a fish recognition model based on CNNs in ubiquitous devices, focusing on the preservation of these species principally during the closed period.
\end{abstract}

\section{Introduction}

Marine biology and aquaculture have reached great importance in recent years [Ding et al. 2017]. At the same time, the recognition of fish has been a relevant topic with the potential for scientific research. Traditionally, marine biologists have identified fish based on their ichthyological characteristics and manually, making the process exhaustive and time-consuming [Ogunlana et al. 2015, Chen et al. 2017]. According to Rodrigues et al., the classification of fish species made by humans is not feasible for practical applications, since it implies high financial expenses, in addition to being vulnerable to errors [Rodrigues et al. 2015].

The recognition of fish is necessary for several reasons, which include extraction of characteristics, determination of behavioral trait, and statistical and quality control of species to be caught. This recognition is also beneficial for population assessments and ecosystem monitoring [Ogunlana et al. 2015]. This practice is also essential for the control of fishing when the existence of a particular species is considered threatened or endangered. According to the 2018 Food and Agriculture Organization (FAO) of the United Nations (UN) technical report, overfishing is a matter of great concern. As the process of rebuilding marine stocks requires time, usually two to three times the life 
of the species 1 . Allken et al. claim that sustainable fish exploitation requires effective management based on continuous monitoring of the environment [Allken et al. 2018]. As a result, it is necessary to realize fisheries control to avoid damage to the exploited species. In the long term, the consequences of excessive fishing activity can be drastic, since fisheries resources contribute to the supply of food. Besides, fishing contributes to job creation in coastal areas.

According to Salman et al., the recognition process involves two stages, to know, fish detection, followed by species classification [Salman et al. 2016]. Fish detection is the process of distinguishing fish from "non-fish" objects, e.g., areas of the boat, human hands holding fish, among others. On the other hand, the classification aims to recognize species. However, classifying and recognizing fish is a complex task, despite its commercial and academic utility. Some of the challenges faced by accurate and reliable fish recognition include to remove distortion, noise, segmentation error, overlapping, and occlusion of digital images [Ding et al. 2017]. Also, solutions for the classification and recognition of fish should consider other issues [Rodrigues et al. 2015]. The first of these is the size of the fish and its, a priori, unknown orientation. Also, there are some attributes that may differ significantly between different species. Another factor is the image acquisition process, which can be affected by noise from various sources, as well as system distortions and anomalies.

In modern computing, the idea that technology is linked to everyday life in the smoothest possible way, capable of adapting to different contexts and capable of solving problems on its own, is called ubiquitous computing [Ocegueda-Miramontes et al. 2019]. In general, ubiquitous systems identify, monitoring, and track users. Besides, most of these systems have at least two of the following built-in features, to know, detection, actuation, network, or decision-making capabilities. A normal workflow in ubiquitous computing usually includes collecting user and context information, the processing of collected data; execution of actions in the corresponding context, and offering information to other systems. Pervasive computing needs techniques that surpass predefined solutions. Thus, there is a need for systems that act intelligently and autonomously [Georgievski] and Aiello 2016]. The artificial intelligence (AI) field focuses efforts on the development of flexible and effective models for smart and autonomous behavior. Among the AI strands, deep learning and convolutional neural networks (CNNs) stand out. According to Hammerla et al., the most successful approach in ubiquitous computing depends on CNNs [Hammerla et al. 2016].

CNNs use several layers in order to extract characteristics from a data set. Currently, this approach has been used in several object recognition tasks, including the recognition of fish species [Jin and Liang 2017]. Through CNNs, it is possible to extract fish dependent characteristics in a unique, invariant, and robust way, despite the distortions and variability of the images. CNNs are a multilayered artificial neural network (ANN) consisting of the input layer, convolutional layer, cluster layer, fully connected layer, and output layer. In this context, the main objective of this study is to propose a model for the recognition of fish at risk of overfishing using a $\mathrm{CNN}$, presenting relative economic importance for coastal communities. The methodology adopted is based on the extraction of images from a data set and analyze them in a CNN-based platform that performs the

\footnotetext{
1 http://www.fao.org/3/I9540EN/i9540en.pdf
} 
classification and recognition. Then, the proposed algorithm is evaluated through various sets of experiments to validate its effectiveness in terms of accuracy.

The paper is organized in sections and Section 2 elaborates on the related work about the topic. The third section justifies the relevance of the study with the theoretical foundation. The used methodology is shown in Section 4 and, in Section 5, the results of the experiments are discussed. Finally, Section 6 presents the conclusion and suggestions for future work.

\section{Related Work}

Several studies have been developed for the classification and recognition of fish. Then, this section discusses recent literature, which is related to the purpose of this research.

Rossi et al. proposed the FishAPP, a mobile application developed for the Android and iOS operating systems. It allows the user to take pictures of an entire fish and upload them to a cloud-based system, which implements complex image processing. Also, the application contains an ANN-based system capable of analyzing the images obtained and performing the classification of predefined fish species [Rossi et al. 2016]. When taking the photo of the fish, the user must follow some rules, to know, the photo must include the whole fish, the fish must be photographed laterally, and the tail fin must be organized anatomically. The authors collected photos from 339 fish samples, divided into seven species. The way the user should photograph the fish limits the application relevance.

In their research, Salman et al. presented a CNN model, which is based on a combination of hierarchical characteristics. These characteristics serve for the model to learn the visual aspects dependent on the fish species that are unique, however, abstract and robust. In this way, the need to extract resources from fish images is avoided using image processing techniques. As a result, the authors obtained a trained model with favorable performance, since the algorithm managed to generalize well even for images of fish species that were not used in training. The reference data sets were LifeCLEF14 and LifeCLEF15. In the end, the result obtained by the authors was a model with a classification rate higher than 90\% [Salman et al. 2016]. One of the restrictions presented by the authors was the CNN training time, which can be optimized using graphics processing units (GPUs).

The research proposed by Manandhar and Burris presents a solution for the identification of common fish in Louisiana's fishing activity in United States of America (USA). The authors used a CNN trained with the machine learning library TensorFlow from Google [Manandhar and Burris 2019]. For the creation of the data set, seven species of saltwater fish commonly found in Louisiana were chosen. The ultimate training precision achieved by the model was $85 \%$, an applicable rate compared to the existing machine learning algorithms.

Given the studies shown in the literature, four aspects are still challenges for the recognition of fish. They are images of fish under controlled conditions; small data sets, which show few images of fish species; inefficiency of algorithms in large data sets, and unsatisfactory accuracy. As a result, the purpose of this paper is to propose a model for recognizing fish at risk of overfishing using a CNN, with support to solve these identified challenges. 


\section{Theoretical Foundation}

The main objective of ubiquitous computing is to be user-centered, supporting people in their everyday life [Meshram et al. 2016]. However, advances in technology, such as wireless communications and mobile devices, imply a rapid evolution of ubiquitous computing environments. Thus, the complexity of these environments also increases, requiring computational techniques to deal with the evolution and uncertainty of the systems dynamically and automatically. AI can contribute to satisfying this scenario in several ways, including the use of CNNs. In this section, the concepts that guided the development of the research proposal are presented.

\subsection{Convolutional Neural Networks}

A CNN is an efficient classification method widely used in object recognition tasks [Jin and Liang 2017]. This approach is composed by a deep learning algorithm that is characterized by having an image as an input and attaches importance to it, called weights, to various objects in the image and is able to differentiate one from the other. In relation to preprocessing, when using a $\mathrm{CNN}$, the time in this step is shorter compared to other classification algorithms. The primitive methods obtain the characteristics manually, while CNNs can learn these characteristics making the process more agile.

The CNN objective is to reduce images in such a way that it is easy to process. However, at the same time, without losing resources that are necessary to obtain a good forecast. This characteristic is essential to design a model with excellent learning resources, and that is scalable for large data sets. In a CNN architecture, the input image passes through a series of processes, which are called layers, in order to obtain an output that allows the image to be classified.

The basic layers of a $\mathrm{CNN}$ are the convolution layers, grouping layers (pooling), and fully connected layers. Figure 1 shows the structure of a CNN. This algorithm receives an image as input. Then, it is subjected to the convolution layer, also called the feature extraction layer, responsible for extracting attributes from the input data. Then, the grouping layer reduces the number of data, retaining useful information, in order to accelerate the training speed. Generally, these two layers work alternately, and the more layers of convolution and grouping, the more resources can be extracted. Finally, in the fully connected layer, high-level attributes are established that correlate with the object class. The output layer shows the species that the fish in the image belongs to.

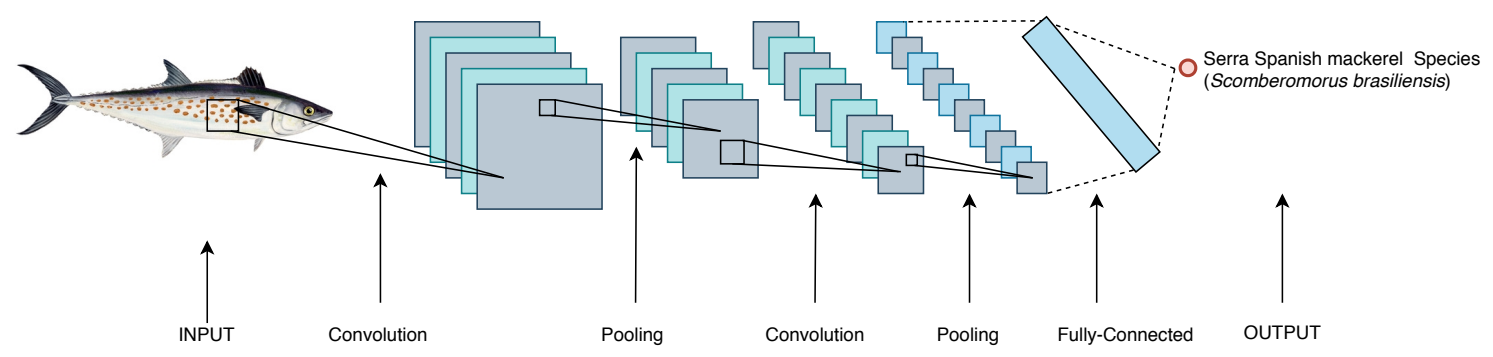

Figure 1. Convolutional Neural Network structure.

A group of application programming interfaces (APIs) has been developed and they aim to facilitate the use of CNNs without the need for automatic learning or experi- 
ence in computer vision. Some APIs are Google Cloud Vision, Clarif.ai, and IBM Watson Visual Recognition. In this paper, the IBM Watson API for image recognition is used.

\subsection{IBM Watson Visual Recognition}

The IBM Watson Visual Recognition $2^{2}$ is a part of the Watson Developer Cloud 3 , built to train models with custom classes based on images provided by the user. This service makes use of deep learning algorithms as a way to analyze images. In them, it can contain scenes, objects, faces and other content. The final answer of the algorithm is given by means of keywords, which provide information about the subject of the images. The IBM Watson visual recognition service provides an accurate and quick classification of the images provided [Aivaliotis et al. 2017].

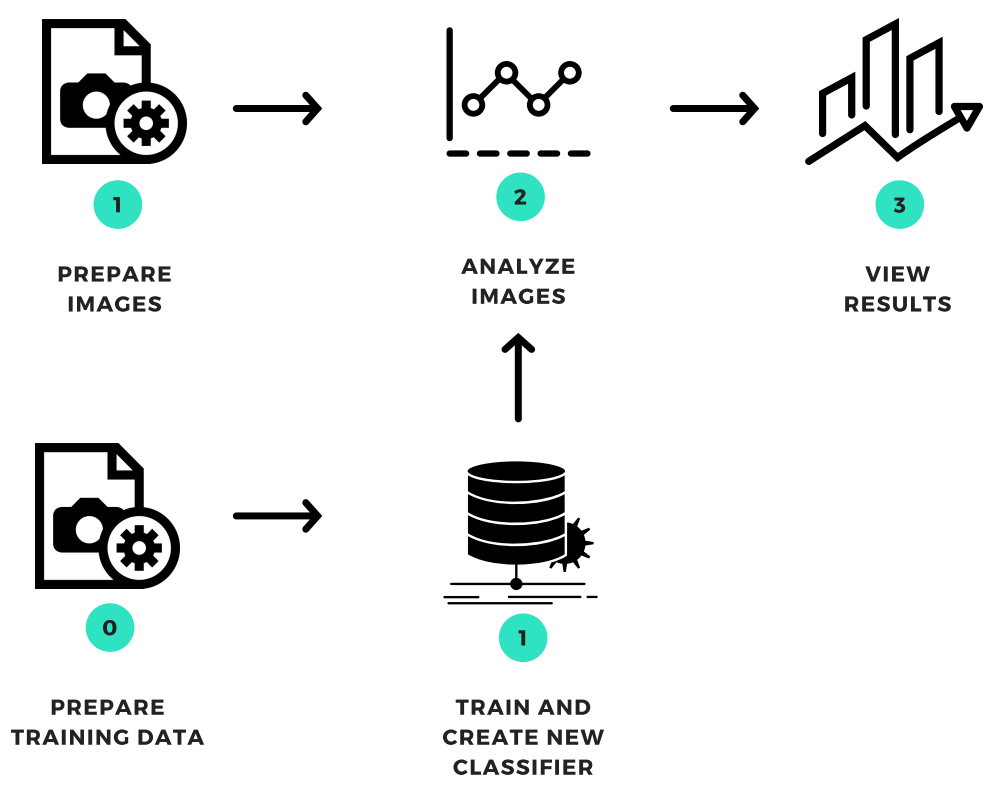

Figure 2. IBM Watson Visual Recognition workflow.

The workflow for the IBM Watson Visual Recognition is shown in Figure2. There are two ways to use this API, to know, using one of the built-in models, or customizing the classifier. Using an embedded model, initially, the images must be prepared to be submitted to the classifier. Aspects such as size and quantity of images must be considered. Then, the images are submitted by the algorithm. Finally, the results are generated and show the classified images. To customize the classifier, the user must prepare data for training. Then, create and train new models. The next steps follow the same flow as the built-in model, i.e., the images are analyzed, submitted to the algorithm, and, finally, the classifier shows the classified images. In this study, the customized model was used to build the classifier, which is detailed in the next section.

2 https://www.ibm.com/watson/services/visual-recognition/ $\sqrt[3]{\text { https://www.ibm.com/br-pt/cloud }}$ 


\section{Materials and Methods}

Ubiquitous computing systems provide access anywhere and anytime to information and various services, in addition to making the system presence as transparent to the user. However, the strategy of obtaining high recognition accuracy at low computational cost is challenging in ubiquitous computing. [Jiang and Yin 2015]. In this way, This section addresses the materials and methods used to deploy the proposed model.

The data set used in this work considers 144 images captured between August and September, 2019. Although the set of images is limited, it was necessary to build a dataset with images close to the reality in which the model could be applied, in order to improve its learning and accuracy. A Canon PowerShot SX170 photographed the images as well as the camera device from a mobile phone iPhone 7 Plus. The images were collected in fish selling establishments in the municipality of Aracati, CE, Brazil, and in the mooring of artisanal fishing vessels existing in that municipality.

The dataset is composed by 12 species of fish commonly found in the Brazilian Northeast. Such species are typical of the coast of this region, in the case of saltwater fish. Figure 3 shows some examples of images from the dataset. These generally include elements other than fish. Some photographs include objects such as people and boats. The selection of images with these variations encourages the model to select identification features that do not depend on the orientation or focus of the image being classified.
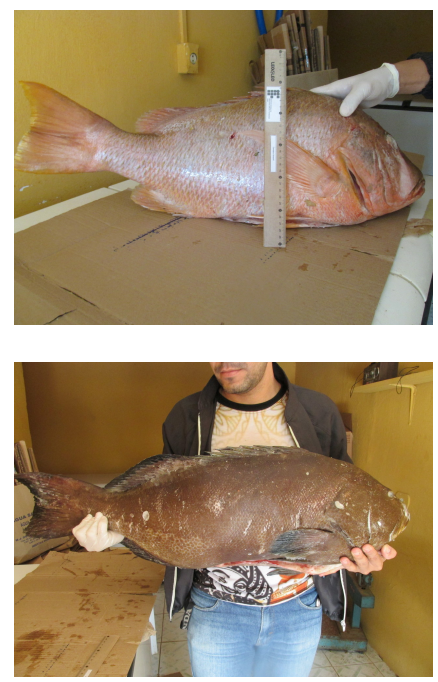
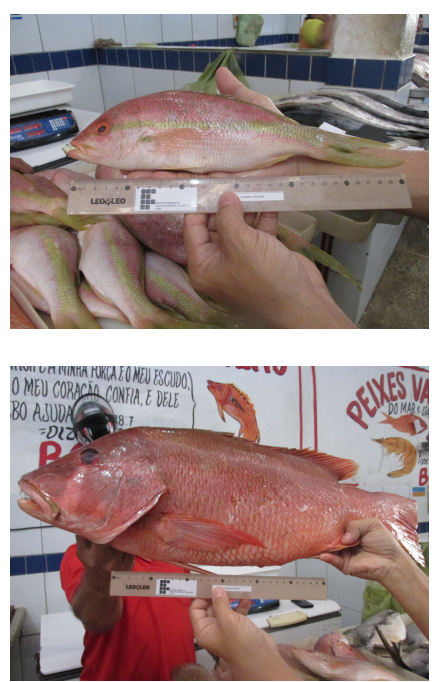
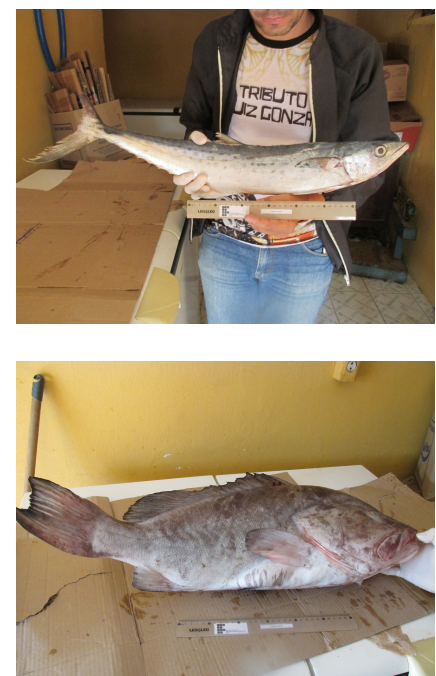

Figure 3. Examples of the dataset images.

The ratio of $80 \%$ to $20 \%$ was used to train and test the algorithm, respectively, i.e., $80 \%$ of the images were used for training and $20 \%$ for testing. This division was due to improving the model processing using more images for training, considering that the images do not contain only the fish as information. The algorithm training has 120 images, 10 images for each class, and 24 images were used to test the algorithm, considering two images per analyzed species. Table 1 shows the distribution of fish species. In this table, species are divided by family. Thus, there is some difficulty to differentiating the characteristic features of species from the same family. As a result, it becomes challenging for the algorithm to differentiate each species. 
Table 1. Species distribution in the dataset.

\begin{tabular}{|c|c|c|}
\hline Popular name & Scientific name & Famíly \\
\hline $\begin{array}{l}\text { Atlantic bonito } \\
\text { Serra Spanish mackerel } \\
\text { Kingfish }\end{array}$ & $\begin{array}{l}\text { Sarda sarda } \\
\text { Scomberomorus brasiliensis } \\
\text { Scomberomorus cavalla }\end{array}$ & Scombridae \\
\hline $\begin{array}{l}\text { Mutton snapper } \\
\text { Yellowtail snapper } \\
\text { Dog snapper } \\
\text { Lane snapper }\end{array}$ & $\begin{array}{l}\text { Lutjanus analis } \\
\text { Lutjanus chrysurus } \\
\text { Lutjanus jocu } \\
\text { Lutjanus synagris }\end{array}$ & Lutjanidae \\
\hline $\begin{array}{l}\text { Yellow jack } \\
\text { Fat snook }\end{array}$ & $\begin{array}{l}\text { Carangoides bartholomaei } \\
\text { Centropomus parallelus }\end{array}$ & Carangidae \\
\hline Black Grouper & Mycteroperca bonaci & Serranidae \\
\hline Smooth weakfish & Cynoscion leiarchus & Sciaenidae \\
\hline Silver king & Tarpon atlanticus & Megalopidae \\
\hline
\end{tabular}

The construction of the model proposed in this paper takes place in two stages, i.e., the fish species classification and the indication of whether or not the fish can be caught. In the first step, the algorithm performed the recognition of fish species. For this, it was necessary to capture the photos of fish. Then, the images were separated by species and then in training and test dataset. For the algorithm, a supervised learning approach was used, to which the training dataset was submitted. The data set was duly divided into folders with the respective species of fish analyzed. Then, the folders corresponding to each species of fish and the training data were submitted and loaded into the IBM Watson Visual Recognition. In the end of the training, which took an average of ten minutes, the IBM Watson generated a model, which made it possible to receive new images of fish, i.e., the test dataset was submitted. Thus, the model was able to classify the image according to the species to which the fish belonged. In the test, the IBM Watson model rated quickly, on an average of one minute. The proposed model used the CNN technique, embedded in IBM Watson Visual Recognition, which assists in intelligent and autonomous decision making, similar to humans.

Next section shows the results obtained from the experiments submitted to the fish species recognition algorithm.

\section{Results Analysis}

The results shown in this section correspond to the experiments using the fish species recognition algorithm.

After collecting images, the dataset was submitted to training through the customized model of IBM Watson Visual Recognition. Then, the test images were submitted to the model. The IBM Watson visual Recognition service provides the accuracy rate for each test image. With these data, the average accuracy by species was generated. Figure 4 shows this average. In the figure, it can be seen that most species had an accuracy rate greater than $70 \%$. Only the species Yellow jack (66\%), Fat snook (50\%), and Kingfish $(30 \%)$ have low performance in this indicator.

Considering the species Kingfish, the model has difficulty in classifying the 
species, as it is from the same family as the Atlantic bonito and Serra Spanish mackerel fish. Thus, the algorithm is confused when classifying species of the same family. The situation is the same for Yellow jack and Fat snook fish because they are from the same family and the customized IBM Watson model tends to misclassify fish.

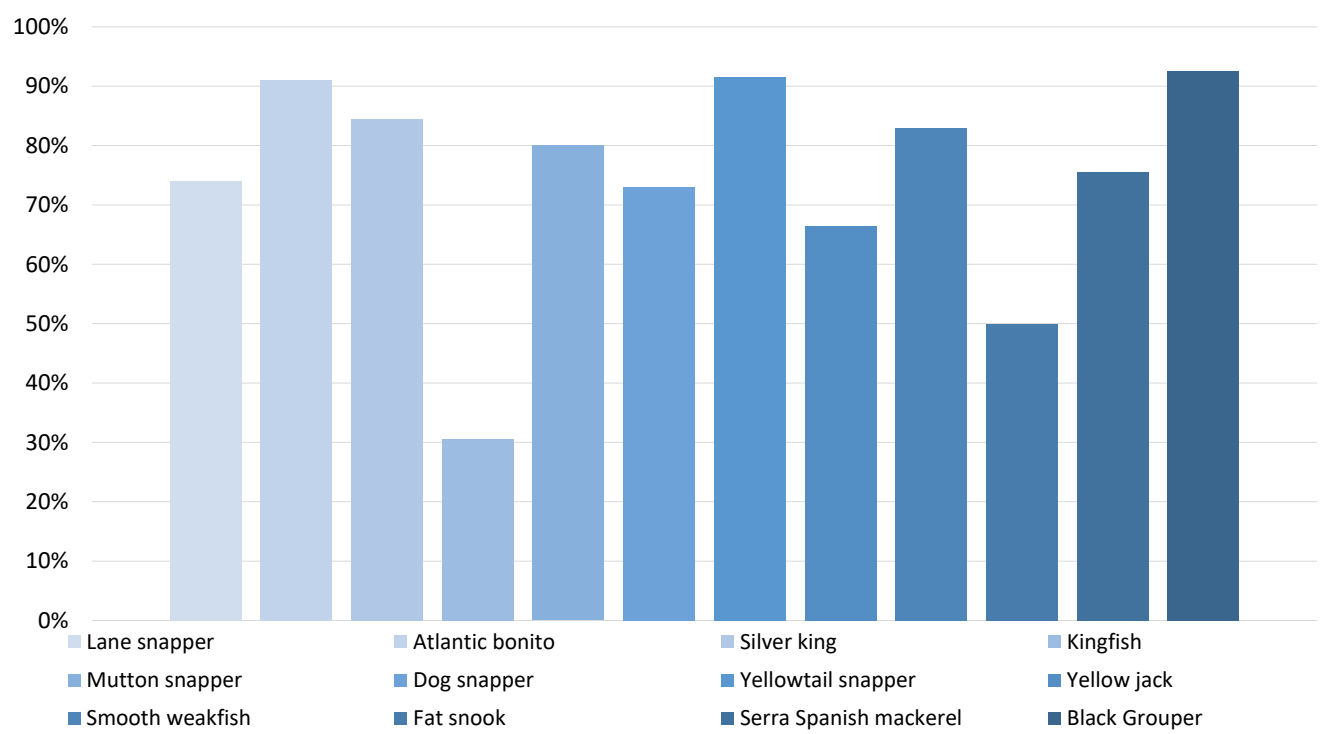

Figure 4. Accuracy by species of fish.

Other results generated are the confusion matrices of the test data set. Figure 5 shows these matrices. They are useful for checking the frequency of hits and errors of the algorithm. The yellow cells represent true-positive values, i.e., the data correctly predicted by the model. The red values correspond to the data mispredicted by the algorithm.

Figure 5(a) shows the confusion matrix considering the species Lane snapper, Atlantic bonito, Silver king, Kingfish, Mutton snapper, and Dog snapper. It shows the algorithm correctly predicted four species. One of the errors occurred in the Lane snapper species, which one of the predicted values corresponded, erroneously, to the Mutton snapper class. It is important to note that Lane snapper and Mutton snapper are species of the same family. The other error presented in this matrix occurred in the species Kingfish. Both values were incorrectly predicted in the Atlantic bonito class. Again, the species Kingfish and Atlantic bonito are from the same family. In Figure 5(b), the confusion matrix corresponds to the species Yellowtail snapper, Yellow jack, Smooth weakfish, Fat snook, Serra Spanish mackerel, and Black Grouper. In it, most species were correctly predicted by the algorithm. Except for the species Fat snook, which one of the values was predicted in the Yellowtail snapper class. The model presents difficulties in distinguishing the species Fat snook from the others.

Finally, the total accuracy of the model was obtained by calculating the accuracy averages by species. Thus, the fish species recognition algorithm had an average rate of $74 \%$ of correct answers. The accuracy of the model reveals that it has good accuracy since it represents that $74 \%$ of the test cases are classified correctly. Thus, the results obtained demonstrate the usefulness of this approach for practical applications in real scenarios of ubiquitous computing. 


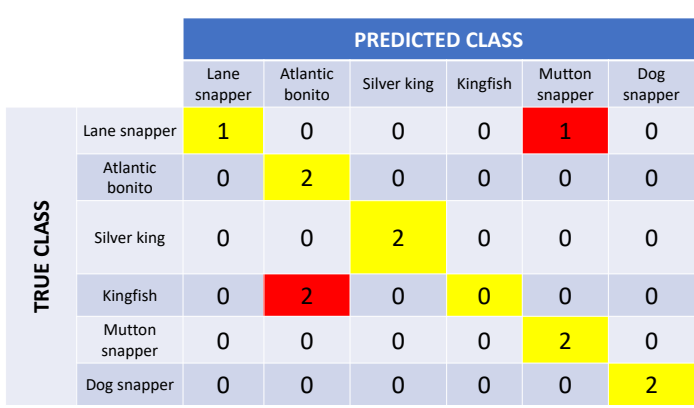

(a)

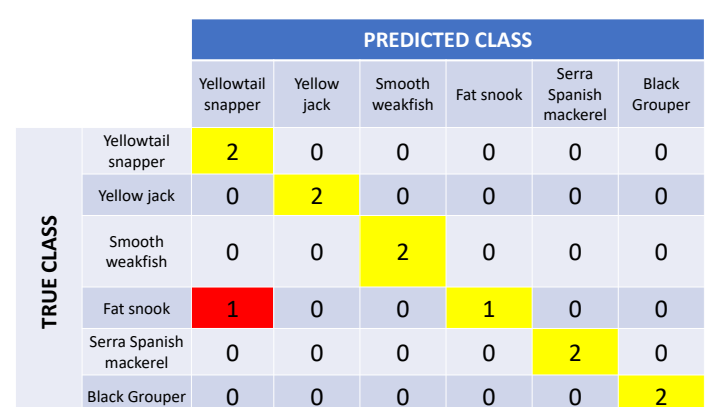

(b)

Figure 5. Confusion Matrices.

\section{Conclusion and Future Works}

This paper presented a fish recognition model for catch control aiming the preservation of marine fish species. The experimental results show the model is capable for recognizing most of the studied fish species, obtaining an accuracy of 74\%. The proposed model reaches the state of the art in terms of recognition accuracy and computational cost, important factors in ubiquitous computing scenarios. This approach proves to be an effective solution for applications related to fish classification and an essential tool for effective fishing control.

The difficulties encountered in carrying out the classification of fish are reflected in the smallest aspects that differentiate species from the same family. Thus, the model's accuracy rate becomes lower. Also, a more significant number of images from the dataset could improve the accuracy of the model and the quality of the statistical analysis.

As further works, it is expected to increase the accuracy of the classification algorithm in order to reach a rate close to $90 \%$. Besides, the algorithm for controlling species capture, i.e., to predict whether or not fish can be caught, are going to be deployed. Also, the objective is to develop a mobile application using the model and subject it to practical scenarios in which it will be possible to test and improve it. Thus, the model can corroborate for fishing resources management, aiming the preservation of fish species.

\section{Acknowledgements}

This work is supported by FCT/MCTES through national funds and when applicable cofunded EU funds under the Project UIDB/EEA/50008/2020; and by Brazilian National Council for Research and Development (CNPq) via Grant No. 309335/2017-5.

\section{References}

[Aivaliotis et al. 2017] Aivaliotis, P., Zampetis, A., Michalos, G., and Makris, S. (2017). A machine learning approach for visual recognition of complex parts in robotic manipulation. In Procedia Manufacturing, volume 11, pages 423-430, Modena, Italy. Elsevier.

[Allken et al. 2018] Allken, V., Handegard, N. O., Rosen, S., Schreyeck, T., Mahiout, T., and Malde, K. (2018). Fish species identification using a convolutional neural network trained on synthetic data. In ICES Journal of Marine Science, volume 76, pages 342349. Oxford University Press. 
[Chen et al. 2017] Chen, G., Sun, P., and Shang, Y. (2017). Automatic fish classification system using deep learning. In 2017 IEEE 29th International Conference on Tools with Artificial Intelligence (ICTAI), pages 24-29, Boston, MA, USA. IEEE.

[Ding et al. 2017] Ding, G., Song, Y., Guo, J., Feng, C., Li, G., He, B., and Yan, T. (2017). Fish recognition using convolutional neural network. In OCEANS 2017-Anchorage, pages 1-4, Anchorage, AK, USA. IEEE.

[Georgievski and Aiello 2016] Georgievski, I. and Aiello, M. (2016). Automated planning for ubiquitous computing. ACM Computing Surveys (CSUR), 49(4):1-46.

[Hammerla et al. 2016] Hammerla, N. Y., Halloran, S., and Plötz, T. (2016). Deep, convolutional, and recurrent models for human activity recognition using wearables. arXiv preprint arXiv:1604.08880.

[Jiang and Yin 2015] Jiang, W. and Yin, Z. (2015). Human activity recognition using wearable sensors by deep convolutional neural networks. In Proceedings of the $23 \mathrm{rd}$ ACM international conference on Multimedia, pages 1307-1310.

[Jin and Liang 2017] Jin, L. and Liang, H. (2017). Deep learning for underwater image recognition in small sample size situations. In OCEANS 2017-Aberdeen, pages 1-4, Aberdeen, United Kingdom. IEEE.

[Manandhar and Burris 2019] Manandhar, N. and Burris, J. W. (2019). An application of image classification to saltwater fish identification in louisiana fisheries. In Proceedings of the 20193 rd International Conference on Information System and Data Mining, pages 129-132, New York, NY, USA. ACM.

[Meshram et al. 2016] Meshram, V., Meshram, V., and Patil, K. (2016). A survey on ubiquitous computing. ICTACT Journal on Soft Computing, 6(2):1130-1135.

[Ocegueda-Miramontes et al. 2019] Ocegueda-Miramontes, V., Sanchez, M. A., and Aguilar, L. (2019). Towards intelligent systems for ubiquitous computing: Tacit knowledge-inspired ubicomp. In Applied Decision-Making, volume 209, pages 6594. Springer.

[Ogunlana et al. 2015] Ogunlana, S., Olabode, O., Oluwadare, S., and Iwasokun, G. (2015). Fish classification using support vector machine. In African Journal of Computing \& ICT, volume 8, pages 75-82. IEEE.

[Rodrigues et al. 2015] Rodrigues, M. T., Freitas, M. H., Pádua, F. L., Gomes, R. M., and Carrano, E. G. (2015). Evaluating cluster detection algorithms and feature extraction techniques in automatic classification of fish species. In Pattern Analysis and Applications, volume 18, pages 783-797. Springer London.

[Rossi et al. 2016] Rossi, F., Benso, A., Di Carlo, S., Politano, G., Savino, A., and Acutis, P. L. (2016). Fishapp: A mobile app to detect fish falsification through image processing and machine learning techniques. In 2016 IEEE international conference on automation, quality and testing, robotics (AQTR), pages 1-6, Kobe, Japan. IEEE.

[Salman et al. 2016] Salman, A., Jalal, A., Shafait, F., Mian, A., Shortis, M., Seager, J., and Harvey, E. (2016). Fish species classification in unconstrained underwater environments based on deep learning. In Limnology and Oceanography: Methods, volume 14, pages 570-585. Wiley Online Library. 